\title{
A phase I study of pexidartinib, a colony-stimulating factor 1 receptor inhibitor, in Asian patients with advanced solid tumors
}

\author{
Jih-Hsiang Lee ${ }^{1}$ Tom Wei-Wu Chen ${ }^{2} \cdot$ Chih-Hung Hsu ${ }^{2,3} \cdot$ Yu-Hsin Yen ${ }^{2} \cdot$ James Chih-Hsin Yang ${ }^{2,3} \cdot$ Ann-Lii Cheng $^{2,3}$ • \\ Shun-ichi Sasaki ${ }^{4}$ LiYin (Lillian) Chiu ${ }^{5}$ - Masahiro Sugihara ${ }^{4} \cdot$ Tomoko Ishizuka $^{4} \cdot$ Toshihiro Oguma $^{4}$. \\ Naoyuki Tajima ${ }^{4} \cdot$ Chia-Chi Lin ${ }^{2,6}$
}

Received: 15 January 2019 / Accepted: 7 February 2019 /Published online: 2 March 2019

(C) The Author(s) 2019

\begin{abstract}
Summary
Background Pexidartinib, a novel, orally administered small-molecule tyrosine kinase inhibitor, has strong selectivity against colonystimulating factor 1 receptor. This phase I, nonrandomized, open-label multiple-dose study evaluated pexidartinib safety and efficacy in Asian patients with symptomatic, advanced solid tumors. Materials and Methods Patients received pexidartinib: cohort 1, $600 \mathrm{mg} / \mathrm{d}$; cohort 2, $1000 \mathrm{mg} / \mathrm{d}$ for 2 weeks, then $800 \mathrm{mg} / \mathrm{d}$. Primary objectives assessed pexidartinib safety and tolerability, and determined the recommended phase 2 dose; secondary objectives evaluated efficacy and pharmacokinetic profile. Results All 11 patients (6 males, 5 females; median age 64, range 23-82; cohort $1 n=3$; cohort $2 n=8$ ) experienced at least one treatment-emergent adverse event; 5 experienced at least one grade $\geq 3$ adverse event, most commonly (18\%) for each of the following: increased aspartate aminotransferase, blood alkaline phosphatase, gamma-glutamyl transferase, and anemia. Recommended phase 2 dose was $1000 \mathrm{mg} / \mathrm{d}$ for 2 weeks and $800 \mathrm{mg} / \mathrm{d}$ thereafter. Pexidartinib exposure, area under the plasma concentration-time curve from zero to $8 \mathrm{~h}\left(\mathrm{AUC}_{0-8 \mathrm{~h}}\right)$, and maximum observed plasma concentration $\left(\mathrm{C}_{\max }\right)$ increased on days 1 and 15 with increasing pexidartinib doses, and time at $\mathrm{C}_{\max }$ $\left(\mathrm{T}_{\max }\right)$ was consistent throughout all doses. Pexidartinib exposure and plasma levels of adiponectin and colony-stimulating factor 1 increased following multiple daily pexidartinib administrations. One patient (13\%) with tenosynovial giant cell tumor showed objective tumor response. Conclusions This was the first study to evaluate pexidartinib in Asian patients with advanced solid tumors. Pexidartinib was safe and tolerable in this population at the recommended phase 2 dose previously determined for Western patients (funded by Daiichi Sankyo; clinicaltrials.gov number, NCT02734433).
\end{abstract}

Keywords Tenosynovial giant cell tumor $\cdot$ Pexidartinib $\cdot$ Pharmacokinetics $\cdot$ Safety $\cdot$ Solid tumors

Chia-Chi Lin

cclin1@ntu.edu.tw

1 National Taiwan University Hospital, Hsin-Chu Branch No. 25, Lane 442, Sec. 1, Jingguo Rd, Hsinchu City 300, Taiwan

2 National Taiwan University Hospital, 7 Chung Shan S Rd, Taipei 10002, Taiwan

3 Graduate Institute of Oncology, National Taiwan University College of Medicine, 7 Chung Shan S Rd, Taipei 10002, Taiwan

4 Daiichi Sankyo Co., Ltd., 1-2-58 Hiromachi, Shinagawa-ku, Tokyo 140-8710, Japan

5 Daiichi Sankyo Co., Ltd., 7F-1, No. 308, Sec. 2, Bade Rd, Zhongshan Dist., Taipei City 104, Taiwan

6 Graduate Institute of Clinical Medicine, National Taiwan University College of Medicine, 7 Chung Shan S Rd, Taipei 10002, Taiwan

\section{Introduction}

Pexidartinib is a novel, orally administered small-molecule tyrosine kinase inhibitor (TKI) with strong selective activity against the colony-stimulating factor 1 receptor (CSF1R) [1]; the receptors KIT and FMS-like tyrosine kinase 3 internal tandem duplication mutation (FLT3-ITD) are also inhibited [2]. Based on these targets, pexidartinib may inhibit tumor growth directly by blocking the oncogenic drivers colonystimulating factor 1 (CSF1), tyrosine protein kinase KIT (cKIT), and FMS-like tyrosine kinase 3 (FLT3) [3-6], or indirectly by modulating the tumor microenvironment and affecting interactions between stromal and tumor cells [7-9]. Pexidartinib may also hinder tumor progression by blocking migration and angiogenesis of the tumor cell [10-12].

The utility of pexidartinib in treating patients with cancer was initially evaluated in a phase I study of Western patients with solid tumors [1]. In the dose-escalation part of the study 
$(n=41)$, grade 3 or higher treatment-related adverse events (AEs) occurred in $11 / 41$ patients $(27 \%)$, and those occurring in more than 1 patient included anemia, increase in aspartate aminotransferase (AST) level, and decrease in lymphocyte count (with each event occurring in 2 patients [5\%]). A total of 8 dose-limiting toxicities (DLTs) occurred in 5 (12\%) patients. The steady-state (day 15) median exposures, maximum observed plasma concentration $\left(\mathrm{C}_{\max }\right)$, and area under the plasma concentration-time curve from 0 to $24 \mathrm{~h}\left(\mathrm{AUC}_{0-24}\right)$ generally increased with increasing dose, and the median time to $\mathrm{C}_{\max }\left(\mathrm{T}_{\max }\right)$ values ranged from 1 to $2 \mathrm{~h}$. In the extension part of study $(n=23)$, pexidartinib treatment resulted in a $52 \%$ overall response rate (ORR) and an $83 \%$ disease control rate by Response Evaluation Criteria in Solid Tumors version 1.1 (RECIST v1.1) in patients with recurrent or inoperable tenosynovial giant cell tumor (TGCT) [1].

This phase I, nonrandomized, open-label multiple-dose study is the first to evaluate the safety and pharmacokinetic profiles of pexidartinib in Asian patients with advanced solid tumors.

\section{Materials and methods}

\section{Patients}

Eligible patients were age 20 or older and had a histologically confirmed solid tumor that had relapsed from or was refractory to standard treatment, or for which standard treatment was not available. Female patients were required to have a negative serum pregnancy test within 14 days prior to treatment allocation, be surgically sterile, or be postmenopausal for $\geq 1$ year; male and female patients of childbearing potential were required to use a highly effective contraception method throughout the study and for up to 90 days after completion. Additional inclusion criteria were Eastern Cooperative Oncology Group (ECOG) performance status of 0 or 1; life expectancy of $\geq 3$ months; adequate hematologic, hepatic, and renal function; resolution ( $\leq$ grade 1 or baseline) of all toxicities from previous cancer therapy; and adequate treatment washout period before registration. Patients were excluded if they had previous use of pexidartinib or any biologic
Fig. 1 Schematic of overall study design and plan. DLT, doselimiting toxicity; MTD, maximum tolerated dose; SMC, safety monitoring committee

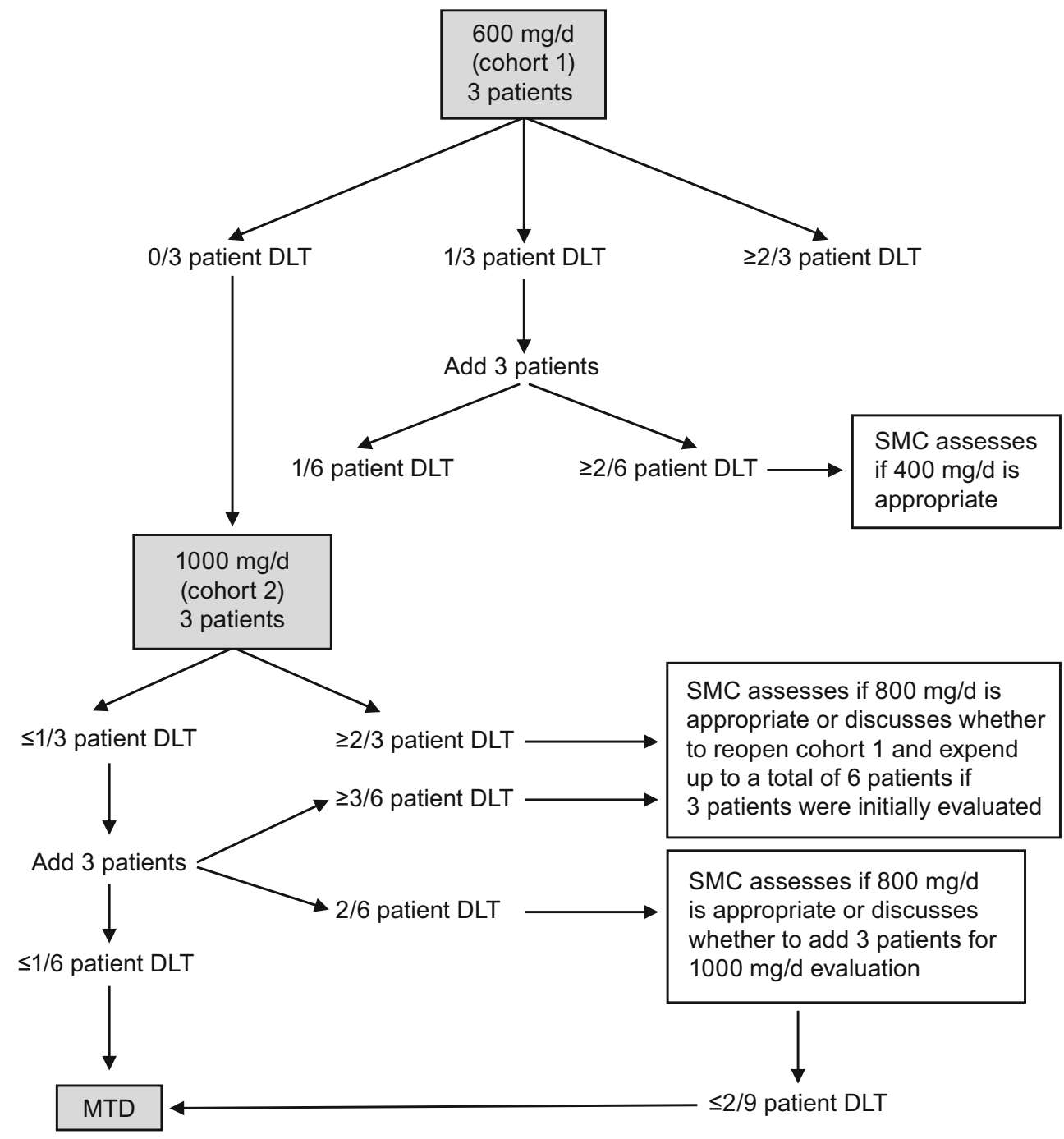


treatment targeting CSF1 or CSF1R, active primary central nervous system tumors or brain metastases, history of lung or heart disease (congestive heart failure, myocardial infarction, or unstable angina), or Fridericia-corrected QT interval (QTcF) $\geq 450 \mathrm{~ms}$ (men) or $\geq 470 \mathrm{~ms}$ (women). In addition, any patients with refractory nausea and vomiting, active or chronic infection with hepatitis $\mathrm{C}$, active tuberculosis, or uncontrolled infection requiring intravenous injection of antibiotics, antivirals, or antifungals were excluded. All patients provided written informed consent.

\section{Study design and treatment}

This phase I, nonrandomized, open-label multiple-dose study was conducted at the National Taiwan University Hospital. The study had a dose-escalation $3+3$ design (June 2016 to June 2017), comprising two dose levels (cohort 1 and cohort 2). Patients in cohort 1 received $600 \mathrm{mg} / \mathrm{d}(200 \mathrm{mg}$ in the morning and $400 \mathrm{mg}$ in the evening); patients in cohort 2 received $1000 \mathrm{mg} / \mathrm{d}$ (400 $\mathrm{mg}$ in the morning and $600 \mathrm{mg}$ in the evening) for the first 2 weeks and $800 \mathrm{mg} / \mathrm{d}$ (400 mg twice per day, in the morning and evening) thereafter (Fig. 1). The decrease in dose from 1000 to $800 \mathrm{mg} / \mathrm{d}$ after 2 weeks was based on a previous phase I study, in which many TGCT patients required dose modification [1]. Each treatment cycle was 28 days. Treatment continued until patient's consent withdrawal, disease progression, or unacceptable toxicity. The maximum tolerated dose (MTD) was determined when no more than 1 of 6 patients experienced a DLT during cycle 1 , which in turn determined the recommended phase 2 dose.

The Institutional Review Board at each participating center approved the study; ethics were in accordance with the Declaration of Helsinki and Good Clinical Practice guidelines of the International Conference on Harmonisation. The study is registered at ClinicalTrials.gov, number NCT02734433.

\section{Study objectives and endpoints}

The primary objectives were to assess the safety and tolerability of pexidartinib and to determine the recommended phase 2

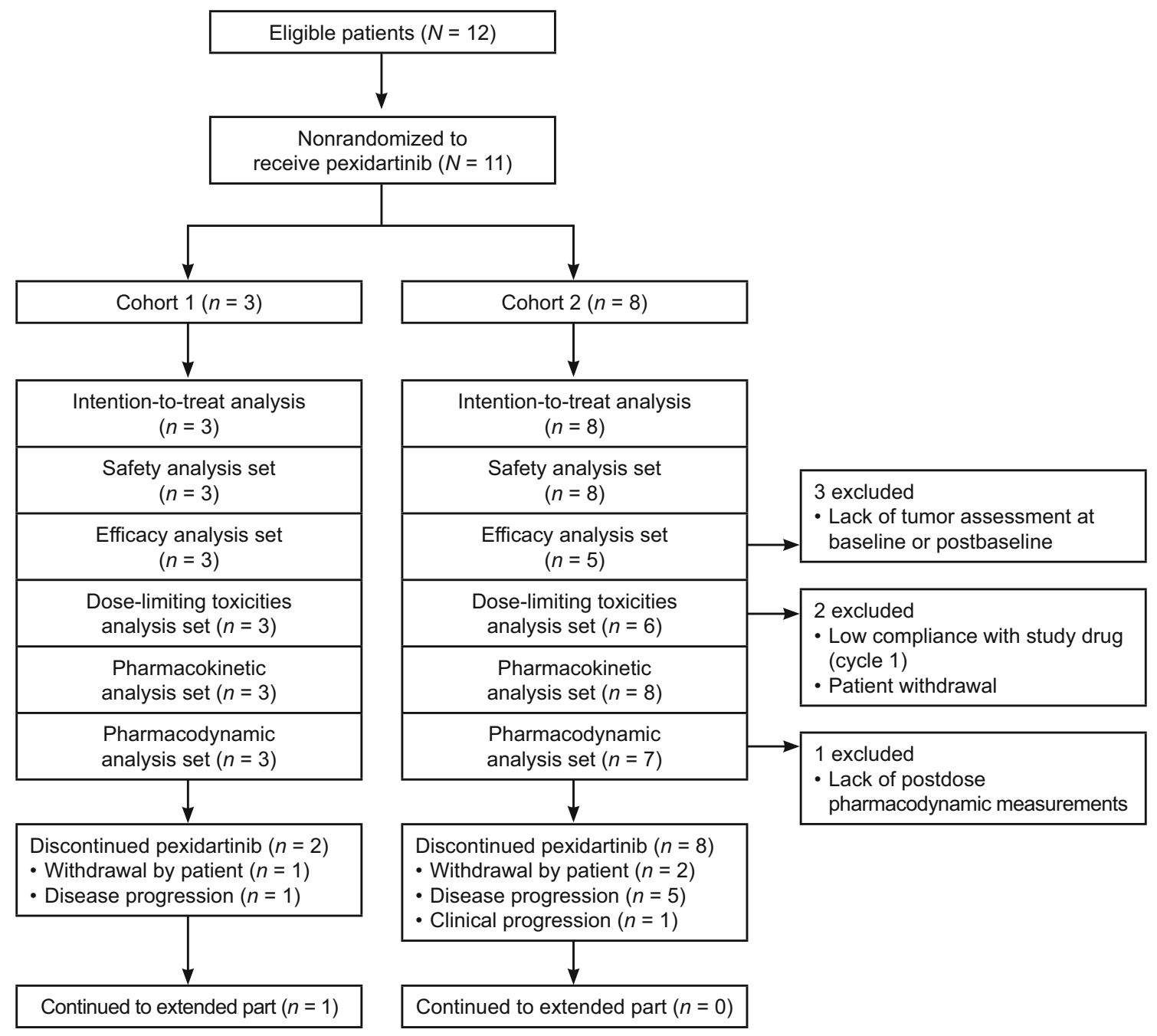

Fig. 2 CONSORT diagram 
dose. The safety endpoints included treatment-emergent adverse events (TEAEs), serious adverse events (SAEs), DLTs, physical examination, vital sign measurements, standard clinical laboratory parameters, electrocardiographic parameters, and echocardiogram/multi-gated acquisition findings.

The secondary objectives were to evaluate the preliminary efficacy, pharmacokinetic profile, and pharmacodynamic effect on plasma levels of CSF 1 and adiponectin of pexidartinib. The efficacy endpoints evaluated tumor response using RECIST v1.1, with the number and percentage of patients in each response category including complete response (CR), partial response (PR), stable disease (SD), progressive disease (PD), time to response, and percentage change in target lesions by magnetic resonance imaging (MRI). Time to response was defined as the time from the date of the first dose to the date at which criteria are first met for CR or PR. Duration of response for responders (CR or PR) was defined as the time interval between the date of the earliest qualifying response and the date of progressive disease or death from any cause, whichever occurs earlier. The pharmacokinetic endpoints evaluated AUC, $\mathrm{C}_{\max }$, and $\mathrm{T}_{\max }$, and the pharmacodynamic endpoints assessed the levels of CSF 1 and adiponectin using plasma collected at various time points after pexidartinib administration. Selection of adiponectin as a marker of PD was based on clinically relevant 2- to 3-fold increases from baseline in patients treated with pexidartinib

Table 1 Patient demographics and baseline disease characteristics

\begin{tabular}{|c|c|c|c|}
\hline Characteristic & $\begin{array}{l}\text { Cohort } 1 \\
n=3\end{array}$ & $\begin{array}{l}\text { Cohort } 2 \\
n=8\end{array}$ & $\begin{array}{l}\text { Total } \\
N=11\end{array}$ \\
\hline Median age (range), year & $68(23-74)$ & $63(40-82)$ & $64(23-82)$ \\
\hline \multicolumn{4}{|l|}{ Sex, $n(\%)$} \\
\hline Male & $2(67)$ & $4(50)$ & $6(55)$ \\
\hline Female & $1(33)$ & $4(50)$ & $5(45)$ \\
\hline \multicolumn{4}{|l|}{ Race, $n(\%)$} \\
\hline Asian & $3(100)$ & $8(100)$ & $11(100)$ \\
\hline \multicolumn{4}{|l|}{ ECOG performance status at baseline, $n(\%)$} \\
\hline 0 & $3(100)$ & $1(13)$ & $4(36)$ \\
\hline 1 & 0 & $7(88)$ & $7(64)$ \\
\hline \multicolumn{4}{|l|}{ Type of solid tumor, $n(\%)$} \\
\hline Bladder cancer, urothelial carcinoma & 0 & $1(13)$ & $1(9)$ \\
\hline Epithelioid trophoblastic tumor & 0 & $1(13)$ & $1(9)$ \\
\hline Gallbladder neuroendocrine carcinoma, large cell type & 0 & $1(13)$ & $1(9)$ \\
\hline Liver cancer & $1(33)$ & 0 & $1(9)$ \\
\hline Malignant fibrous histiocytoma & 0 & $1(13)$ & $1(9)$ \\
\hline Renal cell carcinoma & $1(33)$ & 0 & $1(9)$ \\
\hline Renal pelvic cancer, right; urothelial carcinoma & 0 & $1(13)$ & $1(9)$ \\
\hline Sacral chordoma & 0 & $1(13)$ & $1(9)$ \\
\hline Salivary gland cancer, right submandibular pleiomorphic adenocarcinoma & 0 & $1(13)$ & $1(9)$ \\
\hline Submandibular gland, left; adenoid cystic carcinoma & 0 & $1(13)$ & $1(9)$ \\
\hline Tenosynovial giant cell tumor & $1(33)$ & 0 & $1(9)$ \\
\hline \multicolumn{4}{|l|}{ Prior surgeries, $n(\%)$} \\
\hline 1 & 0 & $1(13)$ & $1(9)$ \\
\hline 2 & $1(33)$ & $3(38)$ & $4(36)$ \\
\hline$\geq 3$ & $2(67)$ & $4(50)$ & $6(55)$ \\
\hline \multicolumn{4}{|l|}{ Prior tumor therapy, $n(\%)$} \\
\hline Yes & $2(67)$ & $7(88)$ & $9(82)$ \\
\hline No & $1(33)$ & $1(13)$ & $2(18)$ \\
\hline \multicolumn{4}{|l|}{ Prior radiation therapy, $n(\%)$} \\
\hline Yes & $2(67)$ & $4(50)$ & $6(55)$ \\
\hline No & $1(33)$ & $4(50)$ & $5(45)$ \\
\hline Concomitant analgesic use, $n(\%)$ & $2(67)$ & $7(88)$ & $9(82)$ \\
\hline
\end{tabular}

ECOG Eastern Cooperative Oncology Group 
Table 2 Summary of adverse events

\begin{tabular}{|c|c|c|c|}
\hline & $\begin{array}{l}\text { Cohort } 1^{\mathrm{a}} \\
n=3 \\
n(\%)\end{array}$ & $\begin{array}{l}\text { Cohort } 2^{\mathrm{b}} \\
n=8 \\
n(\%)\end{array}$ & $\begin{array}{l}\text { Total } \\
N=11 \\
n(\%)\end{array}$ \\
\hline Number of patients with $\geq 1 \mathrm{TEAE}$ & $3(100)$ & $8(100)$ & $11(100)$ \\
\hline Number of patients with $\geq 1$ drug-related TEAE & $3(100)$ & $6(75)$ & $9(82)$ \\
\hline Number of patients with $\geq 1$ serious TEAE & $1(33)$ & $1(13)$ & $2(18)$ \\
\hline Number of patients with $\geq 1$ drug-related serious TEAE & $1(33)$ & 0 & $1(9)$ \\
\hline Number of patients with $\geq 1$ TEAE grade $3 / 4$ & $1(33)$ & $4(50)$ & $5(45)$ \\
\hline Number of patients with $\geq 1$ drug-related TEAE grade $3 / 4$ & $1(33)$ & $2(25)$ & $3(27)$ \\
\hline Number of patients who discontinued due to TEAE & 0 & 0 & 0 \\
\hline Number of patients who died of TEAE & 0 & $1(13)$ & $1(9)$ \\
\hline
\end{tabular}

TEAE treatment-emergent adverse events

${ }^{\text {a }}$ Cohort 1: $600 \mathrm{mg} / \mathrm{d}$ (200 $\mathrm{mg}$ in the morning and $400 \mathrm{mg}$ in the evening)

${ }^{\mathrm{b}}$ Cohort 2: $1000 \mathrm{mg} / \mathrm{d}$ (400 mg in the morning and $600 \mathrm{mg}$ in the evening) for the first 2 weeks. Thereafter, the dose was reduced to $800 \mathrm{mg} / \mathrm{d}$ (400 $\mathrm{mg}$ in the morning and $400 \mathrm{mg}$ in the evening) at dose levels of $900 \mathrm{mg} / \mathrm{d}$ and higher (PLX108-01 US phase I study, unpublished data, 2018).

\section{Study assessments and parameters}

All TEAEs were graded according to the National Cancer Institute Common Terminology Criteria for Adverse Events (NCI-CTCAE) Version 4.03. The DLTs were defined as any drug-related TEAE that occurred during the first 28 days of treatment and met the criteria for hematologic toxicity (grade 4 anemia, neutropenia lasting $>7$ days, or platelet count decrease; grade $\geq 3$ febrile neutropenia or platelet count decrease lasting $>7$ days or associated with bleeding), hepatic toxicity (grade 4 alanine aminotransferase [ALT] or AST increase, ALT or $\mathrm{AST} \geq 3 \times$ upper limit of normal [ULN] if accompanied by $\geq 2 \times$ ULN in total bilirubin, or ALT or AST $>5 \times$ ULN lasting $>14$ days), and nonhematologic toxicity (grade $\geq 3$ nonhematologic, nonhepatic major organ toxicity or inability to complete at least $75 \%$ of the pexidartinib prescribed dose in cycle 1 as a result of a drug-related TEAE).

Efficacy assessments (ORR, disease control rate, duration of response, duration of SD, time to response, and percentage of change in target lesions) were based on tumor assessments completed at screening and at every two cycles ( \pm 7 days) in the first four cycles after cycle 1 day 1 (C1D1) and thereafter at every three cycles ( \pm 7 days) if the patient remained on the study therapy.

A noncompartmental analysis was performed to determine pharmacokinetic parameters including $\mathrm{C}_{\max }, \mathrm{T}_{\max }$, and $\mathrm{AUC}$ from time 0 to $8 \mathrm{~h}\left(\mathrm{AUC}_{0-8 \mathrm{~h}}\right)$ for pexidartinib and its metabolite
Table 3 Grade $\geq 3$ adverse events in any patient or drug-related adverse events in $>1$ patient

\begin{tabular}{|c|c|c|c|}
\hline Preferred term ${ }^{a}$ & $\begin{array}{l}\text { Any grade } \\
N=11 \\
n(\%)\end{array}$ & $\begin{array}{l}\text { Grade } \geq 3 \\
N=11 \\
n(\%)\end{array}$ & $\begin{array}{l}\text { Drug-related } \\
\text { any grade } \\
N=11 \\
n(\%)\end{array}$ \\
\hline Aspartate aminotransferase increased & $5(45)$ & $2(18)$ & $5(45)$ \\
\hline Alanine aminotransferase increased & $4(36)$ & $1(9)$ & $4(36)$ \\
\hline Hair color changes & $4(36)$ & 0 & $3(27)$ \\
\hline Fatigue & $4(36)$ & 0 & $4(36)$ \\
\hline Blood alkaline phosphatase increased & $4(36)$ & $2(18)$ & $4(36)$ \\
\hline Diarrhea & $3(27)$ & 0 & $3(27)$ \\
\hline Gamma-glutamyl transferase increased & $2(18)$ & $2(18)$ & $2(18)$ \\
\hline Anemia & $2(18)$ & $2(18)$ & 0 \\
\hline Blood bilirubin increased & $2(18)$ & $1(9)$ & $1(9)$ \\
\hline Back pain & $2(18)$ & $1(9)$ & 0 \\
\hline Malignant neoplasm progression & $1(9)$ & $1(9)$ & 0 \\
\hline
\end{tabular}

${ }^{\text {a }}$ Classified according to the Common Terminology Criteria for Adverse Events criteria 
Table 4 Summary of TEAE hepatotoxicity laboratory assessment

\begin{tabular}{llll}
\hline Worst value during active treatment period & $\begin{array}{l}\text { Cohort } 1^{\mathrm{a}} \\
n=3\end{array}$ & $\begin{array}{l}\text { Cohort }^{\mathrm{b}} \\
n=8\end{array}$ & $\begin{array}{l}\text { Total } \\
N=11 \\
\end{array}$ \\
& $n(\%)$ & $n(\%)$ & $n(\%)$ \\
\hline $\mathrm{AST} / \mathrm{ALT}>3 \times \mathrm{ULN}$ and $\leq 5 \times \mathrm{ULN}$ & $1(33)$ & $1(13)$ & $2(18)$ \\
$\mathrm{AST} / \mathrm{ALT}>5 \times \mathrm{ULN}$ & $1(33)$ & $1(13)$ & $2(18)$ \\
Total bilirubin $>2 \times \mathrm{ULN}$ & $1(33)$ & $1(13)$ & $2(18)$ \\
$\mathrm{AST} / \mathrm{ALT}>3 \times \mathrm{ULN}$ and concurrent total bilirubin $>2 \times \mathrm{ULN}$ & $1(33)$ & 0 & $1(9)$ \\
\hline
\end{tabular}

$A L T$ alanine aminotransferase, $A S T$ aspartate aminotransferase, $U L N$ upper limit of normal

${ }^{\text {a }}$ Cohort 1: $600 \mathrm{mg} / \mathrm{d}$ (200 mg in the morning and $400 \mathrm{mg}$ in the evening)

${ }^{\mathrm{b}}$ Cohort 2: $1000 \mathrm{mg} / \mathrm{d}$ (400 mg in the morning and $600 \mathrm{mg}$ in the evening) for the first 2 weeks. Thereafter, the dose was reduced to $800 \mathrm{mg} / \mathrm{d}$ ( $400 \mathrm{mg}$ in the morning and $400 \mathrm{mg}$ in the evening)
(ZAAD-1006a). The pharmacodynamic analyses measured plasma levels of the CSF1 and adiponectin biomarkers at predose, cycle 1 day 15 (C1D15), and cycle 2 day 1 (C2D1).

\section{Statistical analysis}

The data cutoff for the primary analysis (June 2017) occurred after all patients either discontinued the study or completed at least four cycles. After the primary analysis, the main study was closed, and the data were followed in the extension part of the study. For the assessment of tumor response, patients were classified, based on their best response, into the following categories: CR, PR, SD, progressive disease, or not evaluable. Exact binomial 95\% confidence intervals (CIs; two-sided) were provided for each category response and the best ORR. The number of nonmissing observations, mean, standard deviation, median, minimum, and maximum as well as geometric means and geometric coefficient of variation were applied for $\mathrm{C}_{\max }$ and AUC pharmacokinetic parameters. The $\mathrm{T}_{\max }$ was summarized using median, maximum, and minimum values.

Assessments of change from baseline to posttreatment or the ratio of posttreatment to baseline included only patients with both baseline and posttreatment measurements. The last nonmissing value of a variable taken before the first dose of study drug was used as the baseline value, unless otherwise specified.

\section{Results}

\section{Patients}

Twelve patients were enrolled from one study center (National Taiwan University Hospital), 11 of whom (6 males and 5 females; median age 64, range 23-82) received pexidartinib; 1 patient was not treated due to a screen failure. Several tumor types were represented in the study, and baseline characteristics
Fig. 3 Percentage change in sum of longest diameters of target lesions from baseline. *The stable disease of this patient is only 53 days after first dosing date, so cannot be considered best overall response. Instead, this patient is classified as having progressive disease

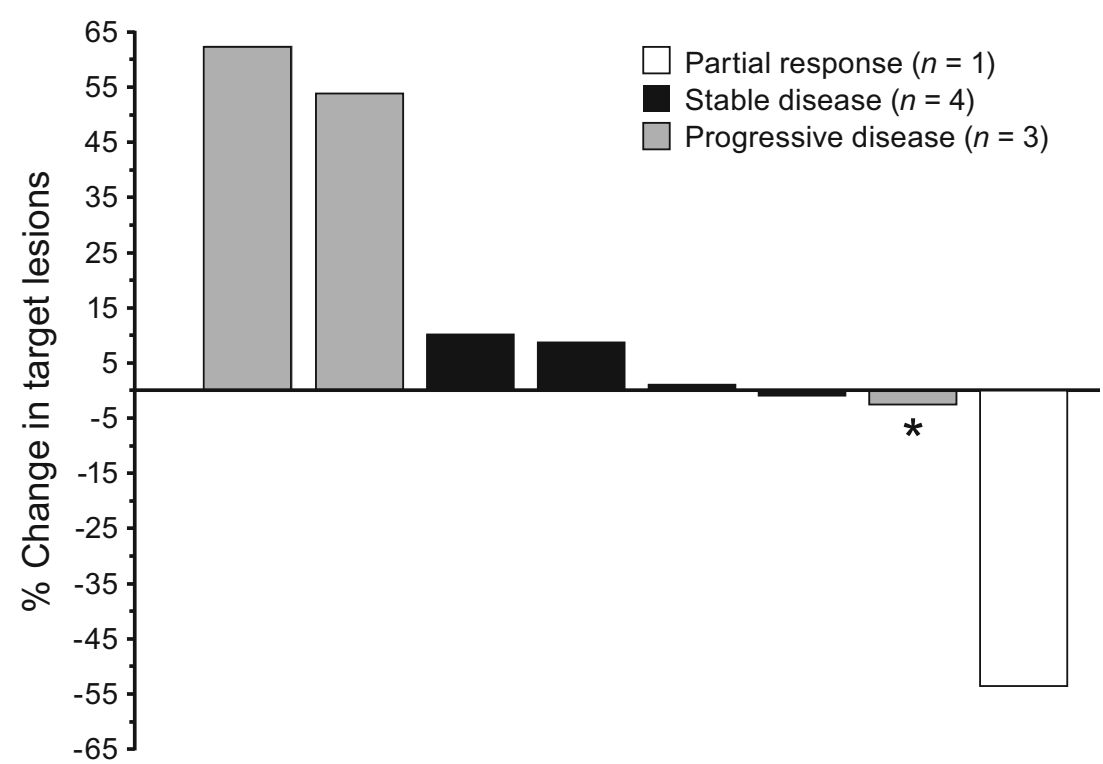


Fig. 4 Longest diameter for right wrist synovial cavity by magnetic resonance imaging

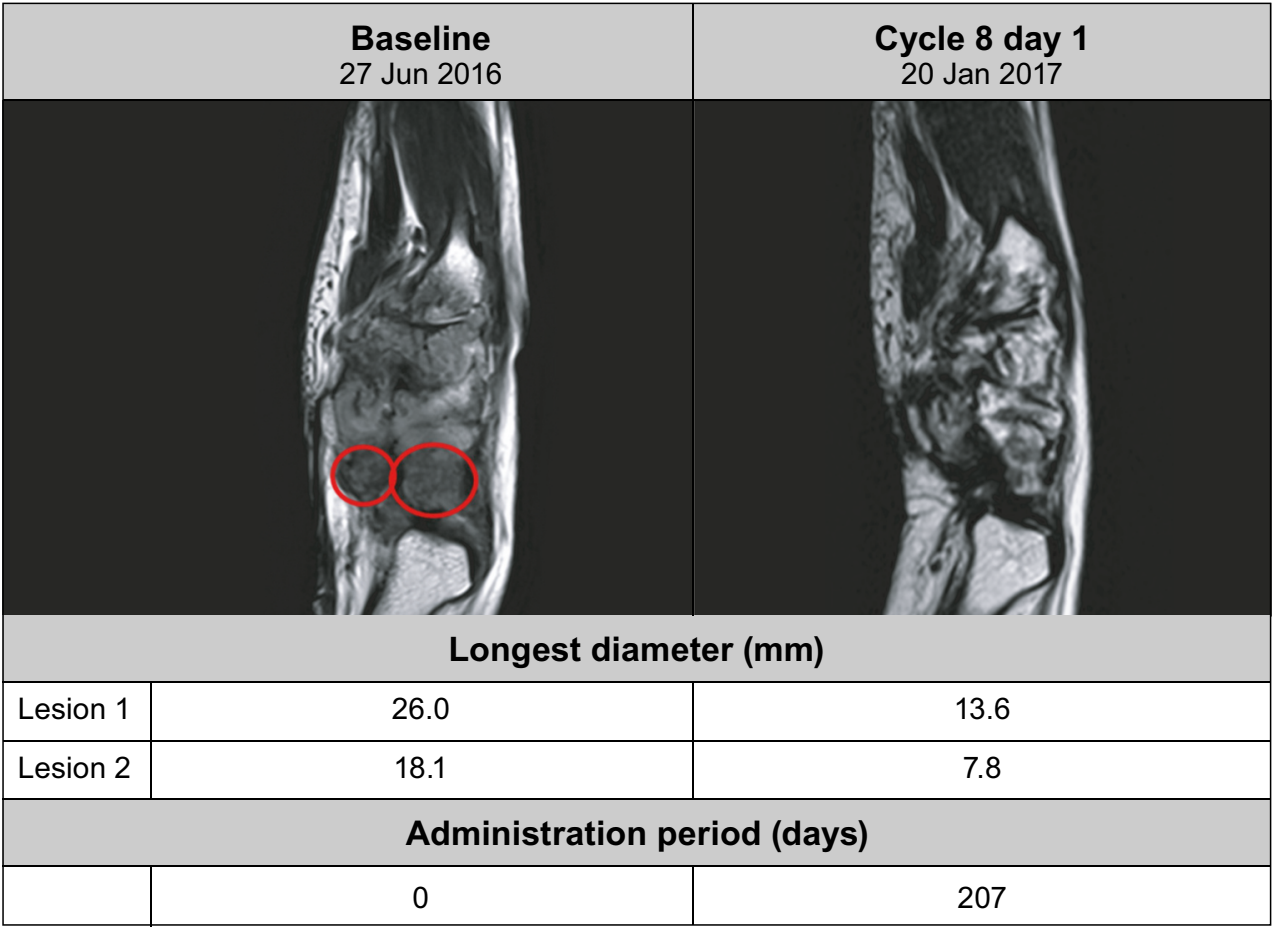

were summarized for cohort $1(n=3$ : renal cell carcinoma, TGCT, liver cancer) and cohort $2(n=8$ : sacral chordoma, malignant fibrous histiocytoma, bladder cancer, epithelioid trophoblastic tumor, submandibular gland, left adenoid cystic carcinoma, salivary gland cancer, large cell gallbladder neuroendocrine carcinoma, renal pelvic cancer) (see CONSORT diagram in Fig. 2). The mean time since diagnosis of solid tumor type was 3.3 years, with a range of 1.7 to 6.6 years. Of the 11 patients, 9 had at least one prior tumor therapy (excluding surgery and radiation therapy) and concomitant analgesic use, while 6 patients had received radiation therapy (Table 1). The TGCT patient from cohort 1 was a 23-year-old female who had prior surgeries (tendon sheath lesion excision and wrist surgery) and prior tumor therapy (oral metronomic cyclophosphamide and prednisolone), but no prior radiation therapy or prior concomitant analgesic use.

\section{Safety}

TEAEs of any grade occurred in all 11 patients (100\%) who received pexidartinib at all dose levels, with 9 $(82 \%)$ of the 11 experiencing a TEAE related to the drug, and $5(45 \%)$ having at least one TEAE of grade 3 or 4 . There was no dose-related trend with drug-related AEs of grade $\geq 3$ (Table 2). The most common TEAEs of any grade were AST increase in 5 patients (45\%) and the following events in 4 patients (36\%) each: ALT increase, fatigue, blood alkaline phosphatase (ALP) increase, and hair color change (Table 3 ). The most common grade 3 or 4 AEs occurred in $18 \%$ of patients each (AST increase, blood ALP increase, gamma-glutamyl transferase increase, and anemia) (Table 3).

Table 5 Summary of pharmacokinetic parameters

\begin{tabular}{|c|c|c|c|c|c|c|}
\hline Cohort & Day & $\begin{array}{l}\mathrm{C}_{\max }(\mathrm{ng} / \mathrm{mL}) \\
\text { geometric } \\
\text { mean }(\% \mathrm{CV})\end{array}$ & $\begin{array}{l}\mathrm{T}_{\max }(\mathrm{h}) \\
\text { median } \\
(\min , \max )\end{array}$ & $\begin{array}{l}\mathrm{AUC}_{0-8 \mathrm{~h}} \\
\left(\mathrm{ng}{ }^{*} \mathrm{~h} / \mathrm{mL}\right) \\
\text { geometric } \\
\text { mean }(\% \mathrm{CV})\end{array}$ & $\begin{array}{l}\mathrm{R}_{\mathrm{Cmax}} \text { geometric } \\
\text { mean }(\% \mathrm{CV})\end{array}$ & $\begin{array}{l}\mathrm{R}_{\mathrm{AUC}} \text { geometric } \\
\text { mean }(\% \mathrm{CV})\end{array}$ \\
\hline \multirow[t]{2}{*}{1} & Cycle 1 day $1(n=3)$ & $3050(40)$ & $2.1(1.8-8.0)$ & $12,800(34)$ & - & - \\
\hline & Cycle 1 day $15(n=3)$ & $8420(16)$ & $1.0(0.0-2.0)$ & $48,900(35)$ & $2.8(47)$ & $3.8(67)$ \\
\hline \multirow[t]{2}{*}{2} & Cycle 1 day $1(n=8)$ & $3460(39)$ & $2.0(0.8-4.0)$ & $15,900(30)$ & - & - \\
\hline & Cycle 1 day $15(n=7)^{\mathrm{a}}$ & $10,400(31)$ & $1.8(0.0-2.1)$ & $62,900(26)$ & $3.2(57)$ & $4.1(52)$ \\
\hline
\end{tabular}

$\mathrm{AUC}_{0-8 \mathrm{~h}}$, area under the plasma concentration-time curve from 0 to $8 \mathrm{~h} ; \mathrm{C}_{\max }$, maximum plasma concentration; \% CV, percent geometric coefficient of variation; $\mathrm{R}_{\mathrm{AUC}}$, ratio of $\mathrm{AUC}_{0-8 \mathrm{~h}}$ on cycle 1 day 15 to $\mathrm{AUC}_{0-8 \mathrm{~h}}$ on cycle 1 day $1 ; \mathrm{R}_{\mathrm{Cmax}}$, ratio of $\mathrm{C}_{\max }$ on cycle 1 day 15 to $\mathrm{C}_{\max }$ on cycle 1 day $1 ; \mathrm{T}_{\max }$, time to reach $\mathrm{C}_{\max }$

${ }^{\text {a }}$ One patient from cohort 2 was excluded from the pharmacokinetic analysis because of a lack of measurements 


\section{a Linear scale}

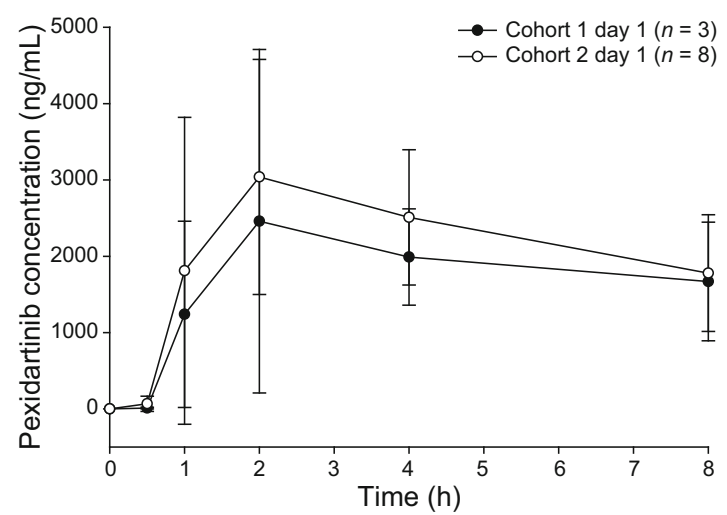

b Semi-logarithmic scale

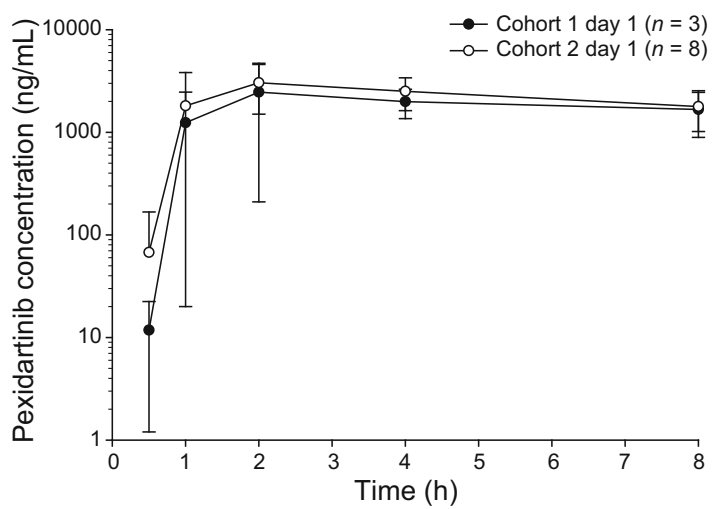

Fig. 5 Mean $( \pm$ STD) plasma concentrations of pexidartinib versus time- day 1. Cohorts 1 and 2: linear (a) and semi-logarithmic (b) scales

Serious TEAEs occurred in 2 of $11(18 \%)$ patients: 1 patient had increased ALT, AST, ALP, and blood bilirubin, and 1 patient experienced anemia as well as malignant neoplasm progression (grade 5), which resulted in death and was considered unrelated to the study drug. Other laboratory parameters that shifted from CTCAE grades $0-2$ to grade 3 postbaseline included: lower lymphocytes $(n=3)$, lower hemoglobin and increased gamma-glutamyl transferase $(n=2)$, lower sodium $(n=1)$, and increased ALP and AST $(n=1)$. There were no changes in vital sign measurements. No DLTs were reported, with the MTD determined to be $1000 \mathrm{mg} / \mathrm{d}$. The recommended phase 2 dose was $1000 \mathrm{mg} / \mathrm{d}$ for the first 2 weeks and $800 \mathrm{mg} / \mathrm{d}$ thereafter.

None of the treatment-related TEAEs in the current study resulted in discontinuation. Two patients ( 1 in each cohort) had the study drug interrupted due to TEAEs, and dose reduction was reported in 1 patient from each cohort. Four patients had treatment-emergent hepatotoxicity laboratory assessment abnormalities: AST/ALT $>5 \times$ ULN, AST/ALT $>3 \times$ ULN and concurrent total bilirubin $>2 \times$ ULN, AST/ALT $>3 \times$ $\mathrm{ULN}$ and AST/ALT $\leq 5 \times \mathrm{ULN}$, and total bilirubin $>2 \times$ ULN (Table 4). Of these abnormalities, only the increased

\section{a Linear scale}

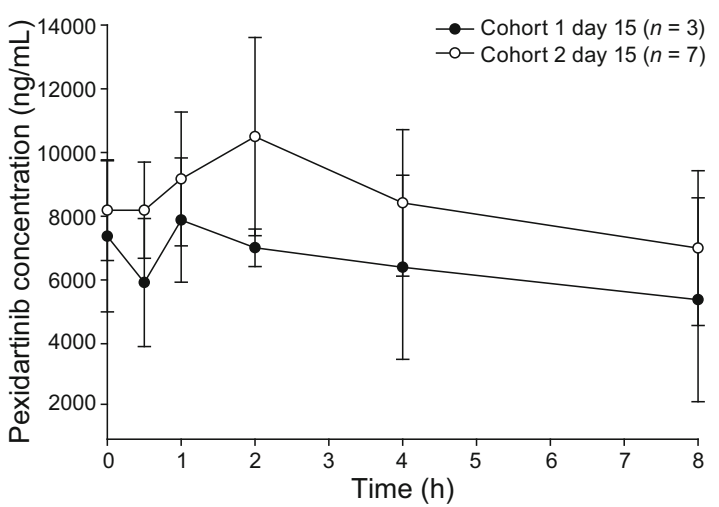

bilirubin remained unrecovered or unresolved and was judged to be unrelated to study drug. None of the patients met Hy's law criteria based on the safety monitoring committee review.

\section{Efficacy}

The overall response rate (CR or PR) by RECIST was $13 \%$, as the PR was found in 1 patient from cohort 1 with TGCT (Fig. 3). This patient continued into the extension part of the study, and nearly 7 months (207 days) into pexidartinib treatment had a large decrease in longest-diameter lesions (lesion 1, from 26.0 to $13.6 \mathrm{~mm}$; lesion 2, from 18.1 to $7.8 \mathrm{~mm}$ ) shown by MRI (Fig. 4). The response was ongoing at 7.6 months in the TGCT patient who completed 13 cycles up to the cutoff date of the dose-escalation part of the study; the patient was still obtaining benefit from the study drug in the extension part of the study. The time to response for the TGCT patient from cohort 1 was 1.9 months. Overall, the disease control rate was $63 \%$ (5/8 patients; $67 \%$ [2/3] in cohort 1 and $60 \%$ [3/5] in cohort 2). There were 4 patients (50\%) with SD with a mean duration of 3.9 months, and $3(38 \%)$ patients with progressive disease.

\section{b Semi-logarithmic scale}

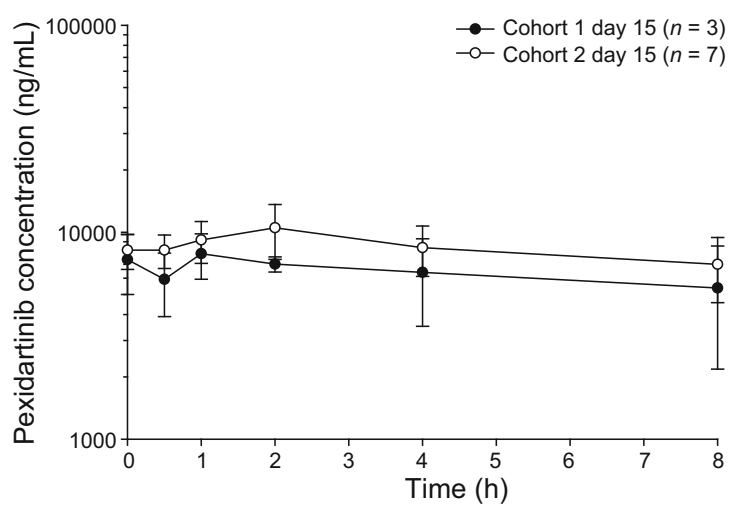

Fig. 6 Mean $( \pm$ STD) plasma concentrations of pexidartinib versus time- day 15. Cohorts 1 and 2: linear (a) and semi-logarithmic (b) scales 
a Linear scale

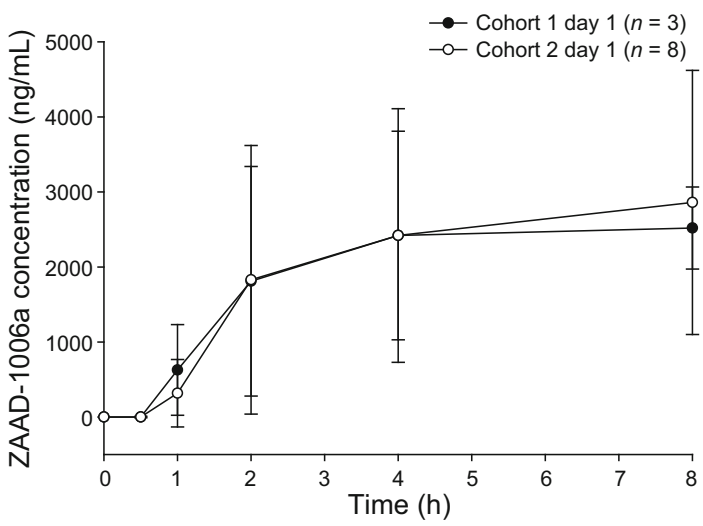

b Semi-logarithmic scale

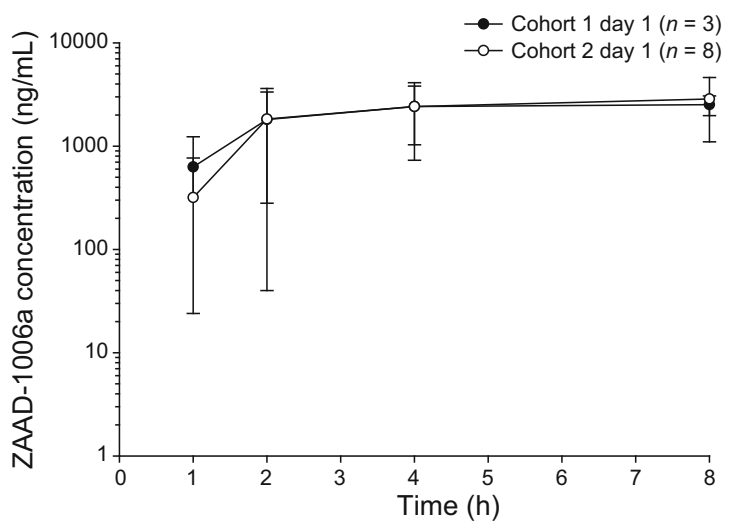

Fig. 7 Mean $( \pm$ STD) plasma concentrations of ZAAD-1006a versus time- day 1. Cohorts 1 and 2: linear (a) and semi-logarithmic (b) scales

The overall mean best percentage change from baseline in the sum of the longest diameters was $9.95 \%$ (range: -53.5 $62.5 \%$ ) (Fig. 3).

\section{Pharmacokinetics and pharmacodynamics}

For the pharmacokinetic analysis in both cohorts, the exposure parameters $\left(\mathrm{AUC}_{0-8 \mathrm{~h}}\right.$ and $\left.\mathrm{C}_{\max }\right)$ increased on days 1 and 15 with increasing doses of pexidartinib, and $\mathrm{T}_{\max }$ was consistent from 600 to $1000 \mathrm{mg} / \mathrm{d}$, with the median ranging from 1.0 to $2.1 \mathrm{~h}$. Pexidartinib plasma concentrations reached steady state within 8 days of twice-daily dose administration based on observation of trough concentrations. $\mathrm{C}_{\max }$ and $\mathrm{AUC}_{0-8 \mathrm{~h}}$ increased approximately 3- and 4-fold, respectively (Table 5). Between cohorts, pexidartinib day 1 exposure (geometric mean $\mathrm{AUC}_{0-8 \mathrm{~h}}$ ) increased by 1.24-fold and geometric mean $\mathrm{C}_{\max }$ increased 1.13fold, with an increase in daily dose from 600 to $1000 \mathrm{mg}$ (Fig. 5 a and b). Day 15 exposure (geometric mean $\mathrm{AUC}_{0-8 \mathrm{~h}}$ ) increased by 1.29-fold and geometric mean $\mathrm{C}_{\max }$ increased 1.24-fold (Fig. $6 \mathrm{a}$ and b). Geometric percent coefficient of variation (\% $\mathrm{CV}$ ) for the pexidartinib exposure parameters ranged from $16.4 \%$ to $39.6 \%$ across the two cohorts and study days.

\section{a Linear scale}

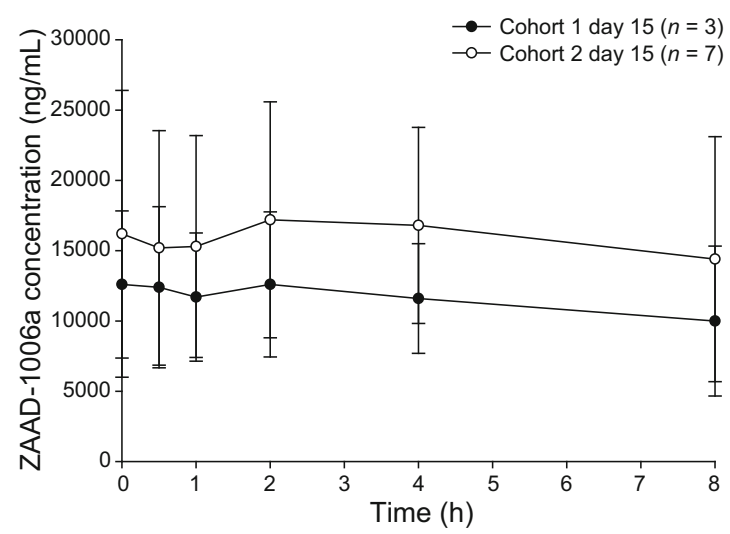

Following multiple oral administrations of pexidartinib, the total exposure $\left(\mathrm{AUC}_{0-8 \mathrm{~h}}\right)$ of the metabolite ZAAD-1006a was approximately 1.2-fold that of the parent drug at day 1 (Fig. $7 \mathrm{a}$ and b) and day 15 (Fig. $8 \mathrm{a}$ and b). Geometric $\% \mathrm{CV}$ for the ZAAD1006a exposure parameters ranged from $35.1 \%$ to $81.6 \%$ across the two cohorts and study days.

In the pharmacodynamic analysis, following multiple-dose administration of pexidartinib, plasma concentrations of CSF1 increased with increasing dose. In cohort 1 , the median CSF1 plasma concentration on C1D1 was $229.83 \mathrm{pg} / \mathrm{mL}$. On C1D15, the median CSF1 increase from baseline was 984.12\%, with no substantial further increase on C2D1 (median 971.51\%) (Fig. 9a). In cohort 2, the median CSF1 plasma concentration on C1D1was $561.02 \mathrm{pg} / \mathrm{mL}$. On C1D15, the median CSF1 increase from baseline was $918.58 \%$, with no further apparent increase on C2D1 (median 627.26\%) (Fig. 9b). In line with the CSF1 findings, plasma concentrations of adiponectin increased with dose following multiple-dose administration of pexidartinib. In cohort 1 , the median adiponectin plasma concentration on C1D1 was $5525.51 \mathrm{ng} /$ $\mathrm{mL}$. On C1D15, the median adiponectin increase from baseline was $146.92 \%$. Adiponectin plasma concentration

\section{b Semi-logarithmic scale}

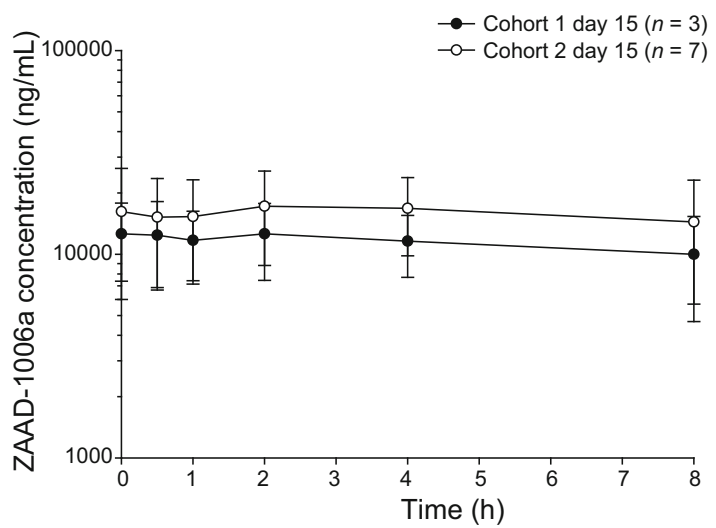

Fig. 8 Mean $( \pm \mathrm{STD})$ plasma concentrations of ZAAD-1006a versus time- day 15. Cohorts 1 and 2: linear (a) and semi-logarithmic (b) scales 
continued to increase on C2D1 (median 320.18\%) (Fig. 9c). In cohort 2, the median adiponectin plasma concentration on C1D1 was $6968.28 \mathrm{ng} / \mathrm{mL}$. On C1D15, the median adiponectin increase from baseline was $99.34 \%$. Adiponectin plasma concentration continued to increase on C2D1 (median 185.91\%) (Fig. 9d). One patient from cohort 2 was excluded from the pharmacodynamic analysis set due to lack of postdose pharmacodynamic measurements.

\section{Discussion}

This phase I, nonrandomized, open-label multiple-dose study was the first to evaluate pexidartinib in Asian patients with advanced solid tumors. Pexidartinib was safe and tolerable, with an MTD of $1000 \mathrm{mg} / \mathrm{d}$ and a recommended phase 2 dose of $1000 \mathrm{mg} / \mathrm{d}$ for the first 2 weeks and $800 \mathrm{mg} / \mathrm{d}$ thereafter, comparable to results from a previous study in Western patients [1]. Pexidartinib treatment resulted in an overall response of $13 \%$ with treatment continuing for over a year. Increases in $\mathrm{AUC}_{0-8 \mathrm{~h}}$ and $\mathrm{C}_{\max }$ were observed with increasing dose of pexidartinib treatment. Pexidartinib exposure increased after

a Cohort $1(n=3 ; 600 \mathrm{mg} / \mathrm{d})$

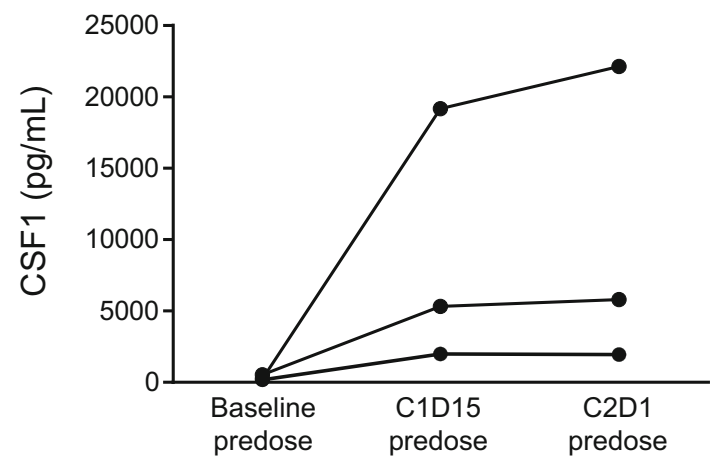

C Cohort $1(n=3 ; 600 \mathrm{mg} / \mathrm{d})$

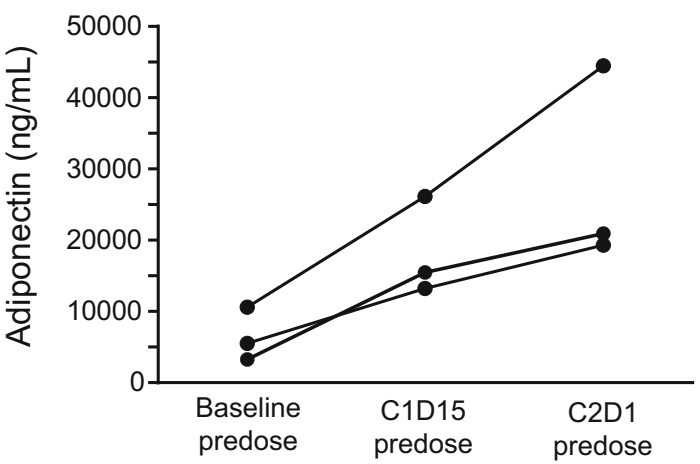

multiple doses, and plasma levels of biomarkers CSF1 and adiponectin increased after pexidartinib administration.

Pexidartinib was generally well tolerated; the most common TEAEs $(\geq 20 \%$ overall incidence) were increased AST, ALT, and blood ALP; fatigue; hair color change; cough; and diarrhea. Five patients experienced grade $\geq 3$ AEs, and there was no dose-related trend with the related drug grade $\geq 3 \mathrm{AEs}$, whereas in the Western population, 11 patients $(27 \%)$ experienced drug-related grade $\geq 3$ AEs [1]. Two patients experienced a total of 6 serious AEs during the dose-escalation phase, one of whom died of malignant neoplasm progression, which the investigator deemed to be unrelated to pexidartinib treatment. There were 4 patients who each experienced one treatment-emergent hepatotoxicity laboratory assessment abnormality. Of those, only 1 patient experienced increased bilirubin that remained unrecovered or unresolved; this was judged to be unrelated to the study drug and did not meet Hy's law criteria as judged by the safety monitoring committee. No DLTs were reported in this study compared to the Western study, in which 5 patients experienced a total of 8 DLTs [1].

In this study, one patient with TGCT showed PR and continued pexidartinib treatment for more than 1 year. This rare

b Cohort $2(n=7 ; 1000 \mathrm{mg} / \mathrm{d}$ for $2 \mathrm{wk}$, $800 \mathrm{mg} / \mathrm{d}$ thereafter)

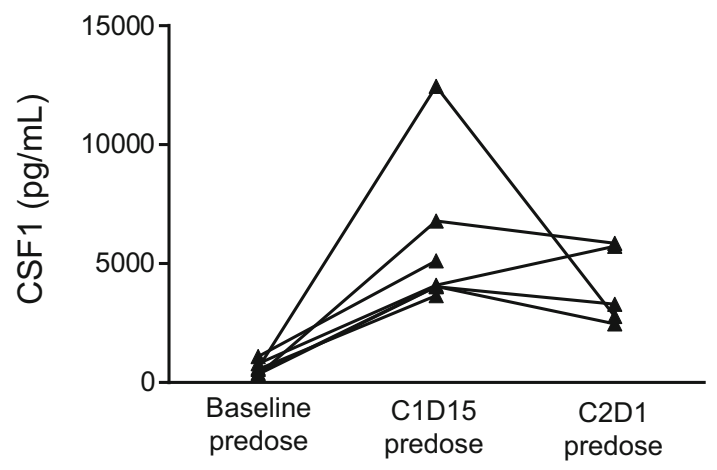

d Cohort $2(n=7 ; 1000 \mathrm{mg} / \mathrm{d}$ for $2 \mathrm{wk}$, $800 \mathrm{mg} / \mathrm{d}$ thereafter)

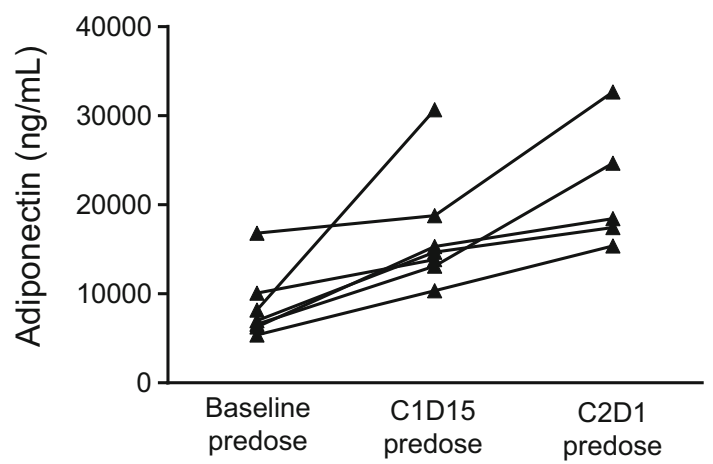

Fig. 9 Individual CSF1 (a-b) and adiponectin (c-d) plasma levels by time. C, cycle; CSF1, colony-stimulating factor 1; D, day 
disease is associated with pain, swelling, limitation of motion, and hemorrhagic joint effusions, which affect quality of life and are clinically important from the patient's perspective [13]. In other studies, treatment with pexidartinib in TGCT patients resulted in an ORR of 52\% (12/23) (phase I) [1] and $39 \%(24 / 61)$ (phase III) [14]. Response rates with other TKI agents have been inferior to the rate observed with pexidartinib. In a retrospective study of advanced TGCT patients treated with imatinib $(n=29)$, ORR was $19 \%$ (phase II) [15], whereas in a single-arm study of nilotinib $(n=56)$, ORR at week 12 was $0 \%$ (phase II) [16].

The current study showed that pexidartinib exposure increased following multiple daily doses of $600 \mathrm{mg}$ (200 and $400 \mathrm{mg}$ ) or $1000 \mathrm{mg}$ ( 400 and $600 \mathrm{mg}$ ), ranging from 3.84 to 4.14 for an accumulation ratio of AUC $\left(\mathrm{R}_{\mathrm{AUC0}-8 \mathrm{~h}}\right)$, and from 2.76 to 3.24 for an accumulation ratio of $\mathrm{C}_{\max }\left(\mathrm{R}_{\mathrm{Cmax}}\right)$. In addition, plasma levels of adiponectin and CSF1 increased following multiple-dose administration of pexidartinib.

Strengths of the study included being the first safety and efficacy evaluation of pexidartinib in Asian patients and demonstrating tolerability and a pharmacokinetic profile comparable to those in previous findings in the Western population [1]. Our result also supports that pexidartinib may offer clinical benefit to TGCT patients. Further studies are needed to assess the role of pexidartinib in solid tumors to determine the optimized dosing schedule and combination regimens. Limitations of the study include the measurement of pharmacokinetic exposure of pexidartinib at $8 \mathrm{~h}$, rather than $24 \mathrm{~h}$ used in the Western population [1]. In addition, only 1 patient achieved a response.

In conclusion, this study further establishes that pexidartinib was safe and tolerable in this population at the recommended phase 2 dose previously determined for Western patients, along with displaying a response in the lone TGCT patient.

Acknowledgments We thank the patients who volunteered to participate in this study; their family members and caregivers; the study center staff members who cared for the patients; the sponsor staff involved in data collection and analyses; and Phillip Giannopoulos, PhD (SciStrategy Communications) for medical writing assistance in the development of the manuscript. Research and manuscript support was provided by Daiichi Sankyo, Co., Ltd.

Funding Sponsored by Daiichi Sankyo, Co., Ltd.; ClinicalTrials.gov number, NCT02734433.

\section{Compliance with ethical standards}

Conflict of interest Ann-Lii Cheng has received remuneration as an officer or advisor from Bristol-Myers Squibb, Ono, Bayer, Merck Sharp \& Dohme, Novartis, and Eisai Corporation and has received honoraria from Novartis, Eli Lilly, AstraZeneca, and Eisai. Shun-ichi Sasaki, LiYin (Lillian) Chiu, Masahiro Sugihara, Tomoko Ishizuka, Toshihiro Oguma, and Naoyuki Tajima have received remuneration as an officer or advisor from Daiichi Sankyo. The other authors have no conflicts of interest.
Ethical approval This study was conducted in compliance with the protocol, the ethical principles that have their origin in the Declaration of Helsinki, the ICH consolidated Guideline E6 for GCP (CPMP/ICH/135/ 95), and applicable local regulatory requirements.

Open Access This article is distributed under the terms of the Creative Commons Attribution 4.0 International License (http:// creativecommons.org/licenses/by/4.0/), which permits unrestricted use, distribution, and reproduction in any medium, provided you give appropriate credit to the original author(s) and the source, provide a link to the Creative Commons license, and indicate if changes were made.

Publisher's note Springer Nature remains neutral with regard to jurisdictional claims in published maps and institutional affiliations.

\section{References}

1. Tap WD, Wainberg ZA, Anthony SP, Ibrahim PN, Zhang C, Healey JH, Chmielowski B, Staddon AP, Cohn AL, Shapiro GI, Keedy VL, Singh AS, Puzanov I, Kwak EL, Wagner AJ, von Hoff DD, Weiss GJ, Ramanathan RK, Zhang J, Habets G, Zhang Y, Burton EA, Visor G, Sanftner L, Severson P, Nguyen H, Kim MJ, Marimuthu A, Tsang G, Shellooe R, Gee C, West BL, Hirth P, Nolop K, van de Rijn M, Hsu HH, Peterfy C, Lin PS, Tong-Starksen S, Bollag G (2015) Structure-guided blockade of CSF1R kinase in tenosynovial giant-cell tumor. N Engl J Med 373(5):428-437

2. Cannarile MA, Weisser M, Jacob W, Jegg AM, Ries CH, Ruttinger D (2017) Colony-stimulating factor 1 receptor (CSF1R) inhibitors in cancer therapy. J Immunother Cancer 5(1):53

3. Fletcher JA, Rubin BP (2007) KIT mutations in GIST. Curr Opin Genet Dev 17(1):3-7

4. Tam WF, Gary Gilliland D (2008) Can FLT3 inhibitors overcome resistance in AML? Best Pract Res Clin Haematol 21(1):13-20

5. Toffalini F, Demoulin JB (2010) New insights into the mechanisms of hematopoietic cell transformation by activated receptor tyrosine kinases. Blood 116(14):2429-2437

6. West RB, Rubin BP, Miller MA, Subramanian S, Kaygusuz G, Montgomery K, Zhu S, Marinelli RJ, de Luca A, Downs-Kelly E, Goldblum JR, Corless CL, Brown PO, Gilks CB, Nielsen TO, Huntsman D, van de Rijn M (2006) A landscape effect in tenosynovial giant-cell tumor from activation of CSF1 expression by a translocation in a minority of tumor cells. Proc Natl Acad Sci U S A 103(3):690-695

7. Dammeijer F, Lievense LA, Kaijen-Lambers ME, van Nimwegen M, Bezemer K, Hegmans JP, van Hall T, Hendriks RW, Aerts JG (2017) Depletion of tumor-associated macrophages with a CSF-1R kinase inhibitor enhances antitumor immunity and survival induced by DC immunotherapy. Cancer Immunol Res 5(7):535-546

8. Pollard JW (2008) Macrophages define the invasive microenvironment in breast cancer. J Leukoc Biol 84(3):623-630

9. Yang FC, Ingram DA, Chen S, Zhu Y, Yuan J, Li X, Yang X, Knowles S, Horn W, Li Y, Zhang S, Yang Y, Vakili ST, Yu M, Burns D, Robertson K, Hutchins G, Parada LF, Clapp DW (2008) Nf1-dependent tumors require a microenvironment containing Nf1+/- and c-kit-dependent bone marrow. Cell 135(3):437-448

10. Coffelt SB, Hughes R, Lewis CE (2009) Tumor-associated macrophages: effectors of angiogenesis and tumor progression. Biochim Biophys Acta 1796(1):11-18

11. Lamprecht B, Walter K, Kreher S, Kumar R, Hummel M, Lenze D, Köchert K, Bouhlel MA, Richter J, Soler E, Stadhouders R, Jöhrens K, Wurster KD, Callen DF, Harte MF, Giefing M, Barlow R, Stein H, Anagnostopoulos I, Janz M, Cockerill PN, Siebert R, Dörken B, 
Bonifer C, Mathas S (2010) Derepression of an endogenous long terminal repeat activates the CSF1R proto-oncogene in human lymphoma. Nat Med 16(5):571-579 571p following 579

12. Steidl C, Lee T, Shah SP, Farinha P, Han G, Nayar T, Delaney A, Jones SJ, Iqbal J, Weisenburger DD, Bast MA, Rosenwald A, Muller-Hermelink HK, Rimsza LM, Campo E, Delabie J, Braziel RM, Cook JR, Tubbs RR, Jaffe ES, Lenz G, Connors JM, Staudt LM, Chan WC, Gascoyne RD (2010) Tumor-associated macrophages and survival in classic Hodgkin's lymphoma. N Engl $\mathrm{J}$ Med 362(10):875-885

13. Staals EL, Ferrari S, Donati DM, Palmerini E (2016) Diffuse-type tenosynovial giant cell tumour: current treatment concepts and future perspectives. Eur J Cancer 63:34-40

14. Tap WD, Gelderblom H, Stacchiotti S, Palmerini E, Ferrari S, Desai J, Bauer S, Blay JY, Alcindor T, Ganjoo KN, Martin Broto J, Ryan CW, Shuster DE, Zhang L, Wang Q, Hsu H, Lin PS, Tong S, Wagner AJ
(2018) Final results of ENLIVEN: a global, double-blind, randomized, placebo-controlled phase 3 study of pexidartinib in advanced tenosynovial giant cell tumor (TGCT). J Clin Oncol 36:abstr 11502

15. Cassier PA, Gelderblom H, Stacchiotti S, Thomas D, Maki RG, Kroep JR, van der Graaf WT, Italiano A, Seddon B, Dômont J, Bompas E, Wagner AJ, Blay JY (2012) Efficacy of imatinib mesylate for the treatment of locally advanced and/or metastatic tenosynovial giant cell tumor/pigmented villonodular synovitis. Cancer 118(6):1649-1655

16. Gelderblom H, Cropet C, Chevreau C, Boyle R, Tattersall M, Stacchiotti S, Italiano A, Piperno-Neumann S, le Cesne A, Ferraresi V, Penel N, Duffaud F, Cassier P, Toulmonde M, Casali P, Taieb S, Guillemaut S, Metzger S, Pérol D, Blay JY (2018) Nilotinib in locally advanced pigmented villonodular synovitis: a multicentre, open-label, single-arm, phase 2 trial. Lancet Oncol 19(5):639-648 